\title{
Emergency resection of brainstem cavernous malformations
}

\author{
*Abdulfettah Tumturk, MD, Yiping Li, MD, Yahya Turan, MD, Ulas Cikla, MD, \\ Bermans J. Iskandar, MD, and Mustafa K. Baskaya, MD \\ Department of Neurological Surgery, University of Wisconsin Medical School, Madison, Wisconsin
}

\begin{abstract}
Brainstem cavernous malformations (CMs) pose significant challenges to neurosurgeons because of their deep locations and high surgical risks. Most patients with brainstem CMs present with sudden-onset cranial nerve deficits or ataxia, but uncommonly patients can present in extremis from an acute hemorrhage, requiring surgical intervention. However, the timing of surgery for brainstem CMs has been a controversial topic. Although many authors propose delaying surgery into the subacute phase, some patients may not tolerate waiting until surgery. To the best of the authors' knowledge, emergency surgery after a brainstem CM hemorrhage has not been described. In cases of rapidly progressive neurological deterioration, emergency resection may often be the only option. In this retrospectively reviewed small series of patients, the authors report favorable outcomes after emergency surgery for resection of brainstem CMs.
\end{abstract}

https://thejns.org/doi/abs/10.3171/2017.1.JNS161693

KEY WORDS brainstem hemorrhage; cavernous malformation; emergency surgery; vascular disorders

$\mathrm{H}$ ISTORICALLY, brainstem cavernous malformations (CMs) have been remarkably challenging to neurosurgeons. Because of their deep-seated location and high surgical risks, conservative therapy has been the mainstay of treatment for many decades. Recently, with advances in our understanding of surgical anatomy, in microsurgical techniques, and in neurophysiological monitoring modalities, overall success in treating what were traditionally deemed "unresectable" lesions has been more promising.

As our understanding of CMs has evolved, we have come to realize that brainstem and other deep-seated CMs may have higher rates of symptomatic hemorrhage and often present with higher rates of neurological compromise compared with their cerebral counterparts. In a recent meta-analysis, the overall estimated annual incidences of hemorrhage and recurrent hemorrhage were $0.3 \%$ and $6.3 \%$, respectively, for non-brainstem CMs compared with $2.8 \%$ and $32.3 \%$ for brainstem CMs. ${ }^{8,15}$ Moreover, the outcomes of untreated brainstem CMs can be poor, as patients with recurrent neurological deficits are less likely to

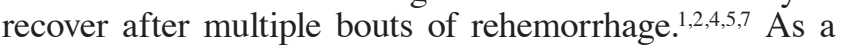

result, there has been debate among those in the neurosurgical field over the optimal treatment strategy.

In young and healthy patients with severe and rapidly progressive deficits we have been aggressive in our management of CMs, especially when surgery appears to be the last resort.

\section{Case Reports}

Case 1

This 23-year-old woman was transferred from an outside facility after being diagnosed with a brainstem hemorrhage on CT (Fig. 1A). She presented with a sudden onset of headaches, left hemiparesis, hemihypoesthesia, dysarthria, double vision, facial paralysis, and ataxia. Upon arrival, MRI was performed that was suggestive of a hemorrhagic neoplasm or brainstem CM (Fig. 1B-D). Over the course of the next several hours after admission, her condition progressed and she became quadriplegic and unresponsive. She underwent intubation and a ventriculostomy was performed. Despite these measures, she stopped having spontaneous respirations, her pupils became pin-

ABBREVIATIONS CM = cavernous malformation; CN = cranial nerve; EMG = electromyography; EVD = external ventricular drain; GCS = Glasgow Coma Scale; GTR = gross-total resection; KPS = Karnofsky Performance Scale; MEP = motor evoked potential; $\mathrm{mRS}=$ modified Rankin Scale; SSEP = somatosensory evoked potential. SUBMITTED July 6, 2016. ACCEPTED January 26, 2017.

INCLUDE WHEN CITING Published online July 7, 2017; DOI: 10.3171/2017.1.JNS161693.

* Drs. Tumturk and Li contributed equally to this work. 

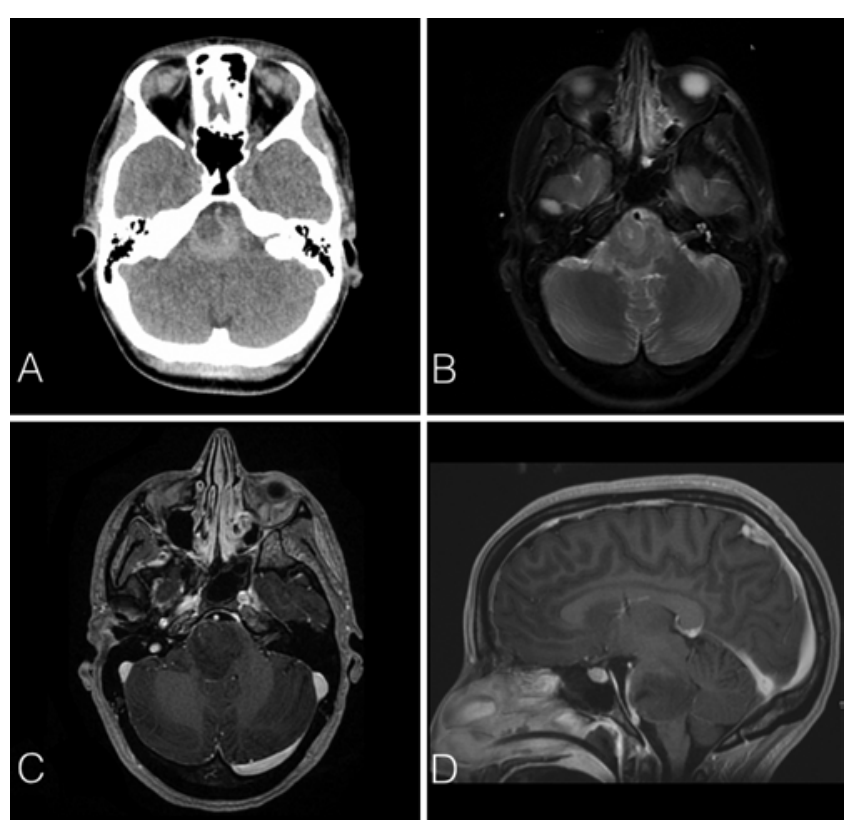

FIG. 1. Case 1. Preoperative axial CT scan (A) and axial T2-weighted MR image (B) show acute and hyperacute phases of hemorrhage in the pons. Axial T1-weighted postcontrast MR image (C) shows minimal enhancement within the lesion at the level of the internal acoustic canal. Sagittal T1-weighted postcontrast MR image (D) shows an expansile mass in the pons with significant mass effect on the basilar artery.

point and sluggishly reactive, and she lost bilateral corneal reflexes.

She was taken emergently to the operating room. Facial nerve monitoring was used (with a nerve integrity monitor) as we performed a right-sided retrosigmoid craniotomy approaching the lateral aspect of the pons. After mapping the facial nerve exit zone, entrance to the pons was made ventral to cranial nerves (CNs) VII and VIII. The lesion was visually consistent with a brainstem CM intraoperatively and was therefore resected. Postoperative MRI demonstrated gross-total resection (GTR) and improvement of mass effect (Fig. 2). On postoperative Day 1, she was following commands and her strength had drastically improved on the right side. Because she lost her gag reflex before surgery and prolonged intubation was anticipated, she underwent elective tracheostomy. She continued to improve to the point that she was ultimately discharged to rehabilitation. Upon her last follow-up evaluation in the clinic after 18 months, she had residual House-Brackmann Grade III facial weakness, partial CN VI palsy, 4/5 motor strength in the left lower extremity, 3/5 in the left upper extremity, and was ambulating independently with a cane.

\section{Case 2}

This 34-year-old man was transferred from an outside facility after receiving a diagnosis of pontomesencephalic junction hematoma. He had a previous known history of a brainstem CM with multiple previous hemorrhagic episodes, resulting in $\mathrm{CN}$ deficits. MRI was performed at an outside facility, which demonstrated obstructive hydrocephalus with a mixed solid and cystic infratentorial lesion centered in the pontomesencephalic junction and extending into the right cerebral peduncle and fourth ventricle (Fig. 3). Upon arrival to our facility his pupils were bilaterally fixed and dilated. He had no cough or gag response, and no corneal reflex or doll's eyes reflex. There was no reaction to noxious stimulation and no spontaneous respirations were observed. After an emergency ventriculostomy his pupillary light and corneal reflexes returned.

The patient underwent emergency surgical evacuation through a supracerebellar infratentorial approach. Somatosensory evoked potentials (SSEPs), motor evoked potentials (MEPs), and facial nerve electromyography (EMG) were used continuously for intraoperative monitoring. Hemorrhagic lesions were visualized on the surface of the pontomesencephalic junction. The lesion was consistent with a CM and was gross-totally removed. Postoperative MRI demonstrated GTR and improvement of mass effect (Fig. 4). On postoperative Day 2, the patient began following commands. He eventually required ventriculoperitoneal shunt placement. After a prolonged hospital course, he was discharged to rehabilitation. At the last follow-up, 54 months postoperatively, he demonstrated House-Brackmann Grade II facial weakness and very mild hemiparesis on the left side, but was ambulating independently.

\section{Case 3}

This 24-year-old man was transferred from an outside facility after being diagnosed with a ventral pontine hemorrhage. He had been complaining of severe headaches for more than 1 week prior to presenting acutely with right-sided facial palsy, anisocoria, and right arm weakness. MRI performed at an outside hospital demonstrated
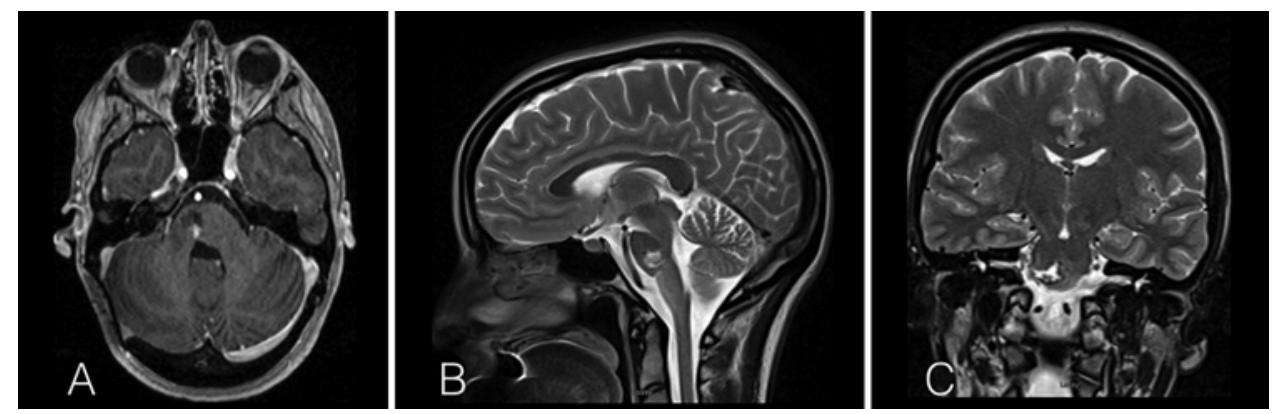

FIG. 2. Case 1. Postoperative axial T1-weighted postcontrast (A), sagittal T2-weighted (B), and coronal T2-weighted (C) MR images show no obvious residual CM and significant improvement of mass effect. 

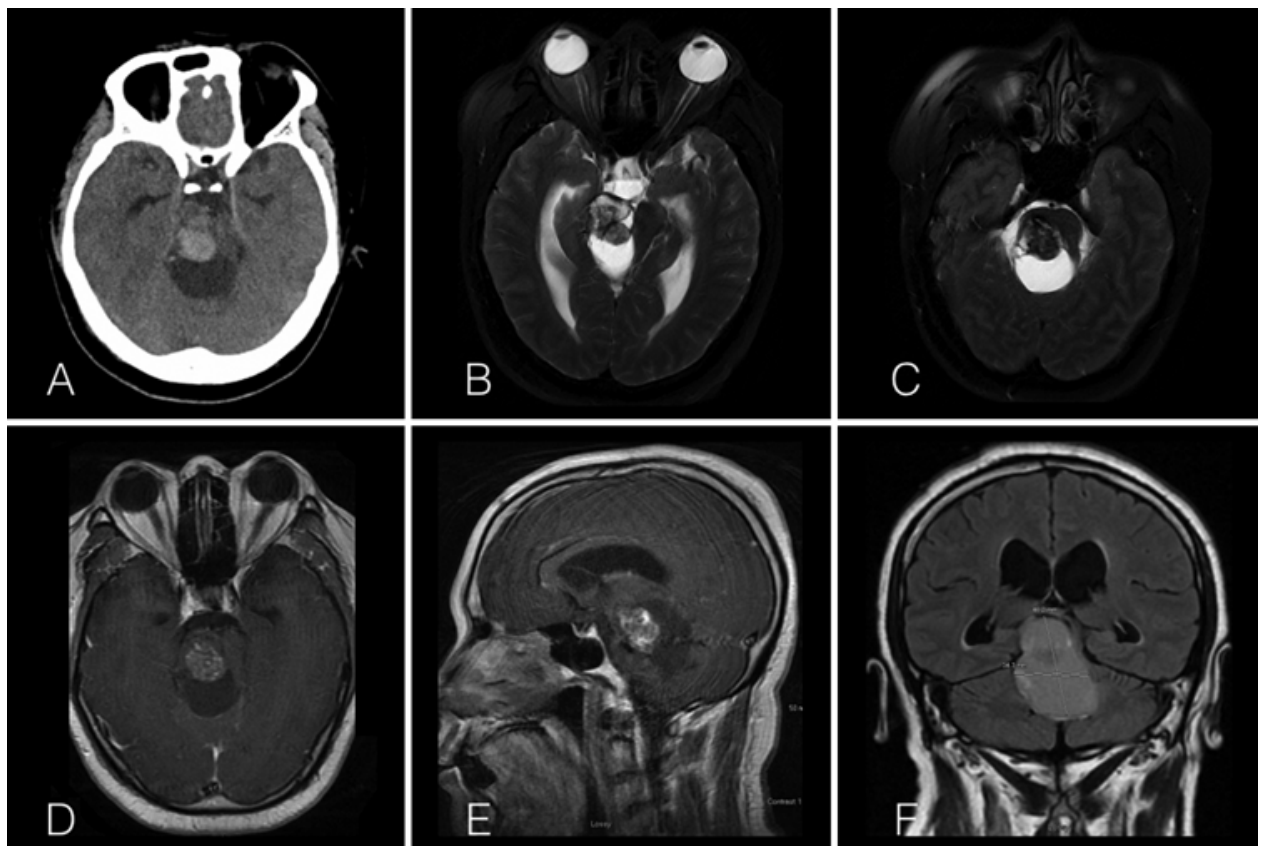

FIG. 3. Case 2. Preoperative axial CT scan (A) and axial T2-weighted MR images (B and C) show acute and hyperacute phases of hemorrhage in the midbrain, pons, and right cerebral peduncle with a cystic component and obstructive hydrocephalus. Axial T1-weighted postcontrast (D), sagittal T1-weighted postcontrast (E), and coronal T2-weighted FLAIR (F) MR images show a heterogenic enhancing mass involving the pons and the right cerebral peduncle extending into the fourth ventricle, as well as the anterior pons.

a ventral pontine hemorrhage extending into the midbrain with extensive surrounding edema (Fig. 5). Soon after arrival, he developed quadriplegia and lost gag reflexes on both sides; therefore, the decision was made to treat this CM emergently.

An emergent external ventricular drain (EVD) was placed and the patient underwent emergency surgical evacuation through a left retrosigmoid craniotomy. Facial nerve EMG, SSEPs, and MEPs were used continuously for intraoperative monitoring. There was no visible discoloration on the surface of the pons. Therefore, a small incision was made between the fifth and seventh $\mathrm{CN}$ zones in the pons, after mapping the facial nerve exit zone with a facial nerve stimulator. The lesion was consistent with a CM and was gross-totally removed. Postoperative MRI demonstrated GTR and improvement of mass effect (Fig. 6). After surgery he significantly improved, with return of his left-sided motor functions first. He continued to im- prove and was discharged to rehabilitation. Upon his last follow-up after 24 months, he had residual mild dysarthria and mild paresis of the right upper extremity.

\section{Case 4}

This 3-year-old girl presented with new-onset ataxia. She had developed left facial weakness 3 weeks prior and was diagnosed with Bell's palsy, which was managed conservatively without further imaging at an outside hospital. Her facial weakness had improved slowly since the acute onset but now had developed new ataxia resulting in multiple falls. She presented to the emergency room, where MRI was performed that revealed a large mixed solid and cystic infratentorial lesion centered in the left pons (Fig. 7).

Because of the development of new and severe ataxia and worsening neurological examination, the patient underwent surgical evacuation through a left retrosigmoid
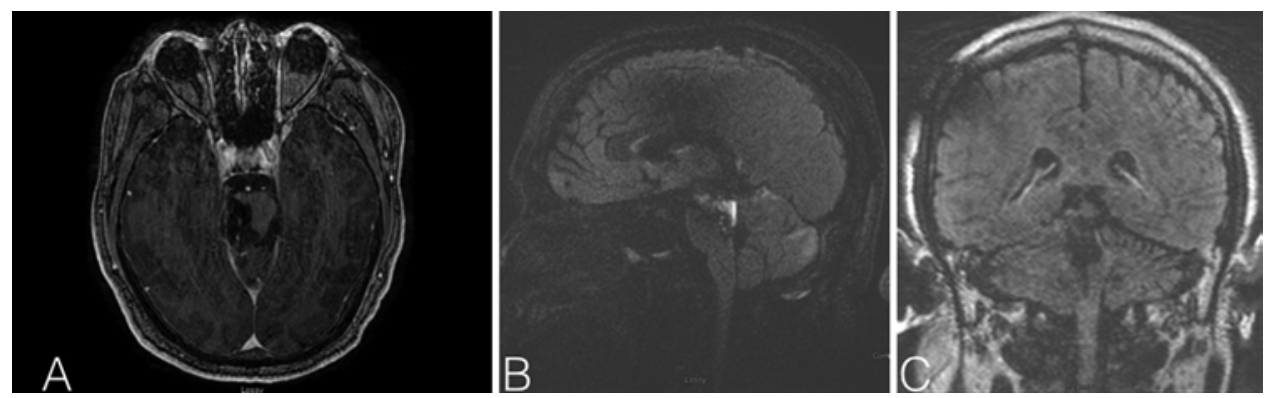

FIG. 4. Case 2. Postoperative axial T1-weighted postcontrast (A), sagittal T2-weighted CUBE-FLAIR (B), and coronal T2-weighted CUBE-FLAIR (C) MR images show no obvious residual CM and significant improvement of mass effect. 

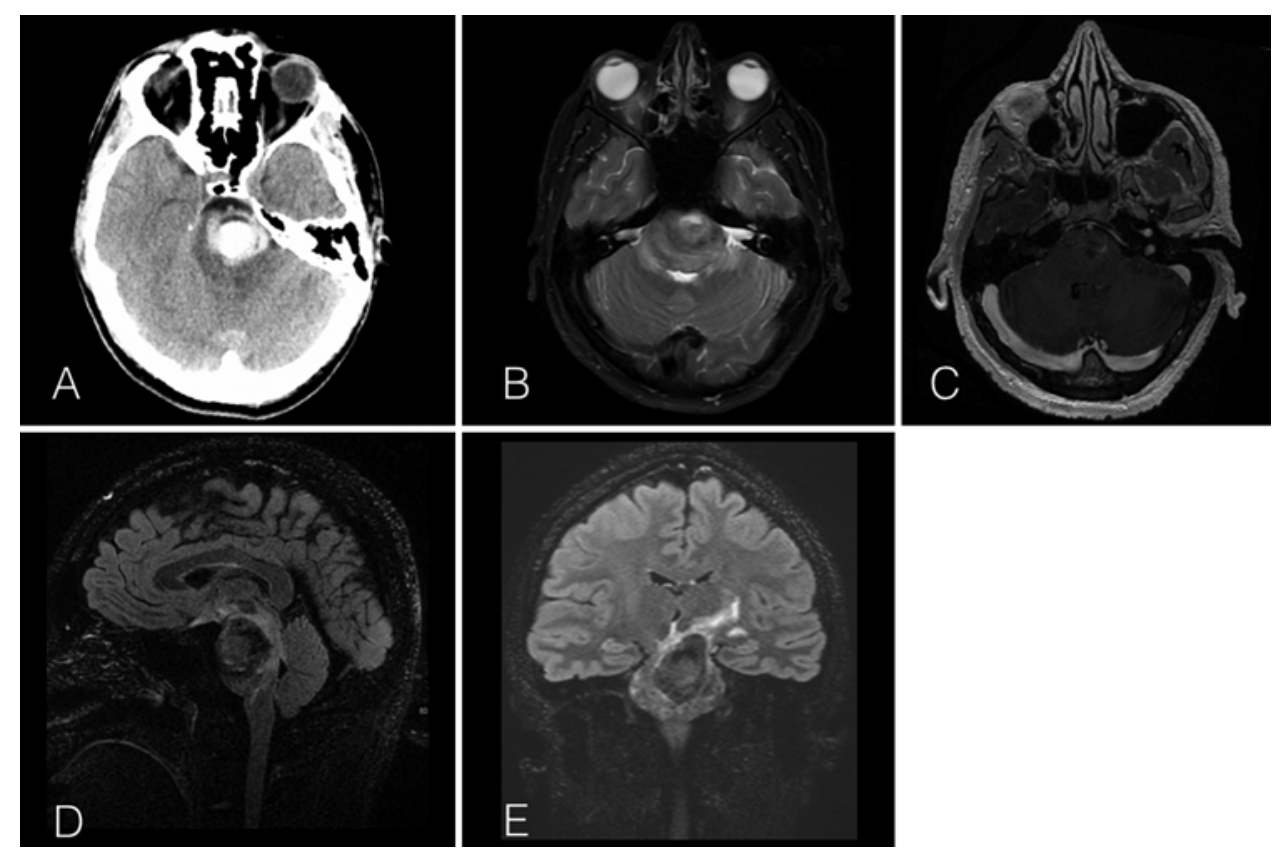

FIG. 5. Case 3. Preoperative axial CT scan (A) and axial T2-weighted MR image (B) show a hemorrhagic lesion involving the pons and left cerebral peduncle. An axial T1-weighted postcontrast MR image (C) shows a minimally enhancing lesion in the pons. Sagittal T2-weighted (D) and coronal T2-weighted CUBE-FLAIR (E) MR images show an expansile mass in the pons with significant mass effect.

craniotomy and intraoperative placement of an EVD emergently. Intraoperative facial EMGs, SSEPs, and MEPs were used for monitoring. Postoperative MRI demonstrated GTR and improvement of mass effect (Fig. 8). She was discharged home with outpatient therapies. Two days after discharge, she presented back to the emergency room with leakage through the previous EVD suture removal site. This leak was repaired at the bedside and the patient was discharged without further complications. At the last follow-up, after 66 months, she had residual mild facial weakness (House-Brackmann Grade II) but complete resolution of ataxia.

\section{Discussion}

An old adage in neurosurgery states that neurological status prior to treatment predicts postoperative neurological recovery. This axiom holds true for many neurosurgical pathologies, with the only consistent exception to the rule being patients presenting with acute neurological decline due to acute compressive mass lesions. One example of such an exception can be observed after surgical decompression of epidural hematomas, when dramatic improvements in neurological function are frequently observed. The outcomes in our case series also appear to fit into the category of exceptions to the rule (Table 1).

The cases presented illustrate the potential value of emergency extirpative surgery in patients with symptomatic brainstem CMs presenting with rapidly progressive neurological deterioration. The frequency of fatal or progressive brainstem CM hemorrhages remains unknown. Traditionally, CT imaging was the modality of choice for brainstem hematomas; therefore, many fatal CM cases could have been missed.

Spontaneous brainstem hemorrhages are a frequent cause of death worldwide. Recent studies report that the overall mortality rate after a primary brainstem hemor-
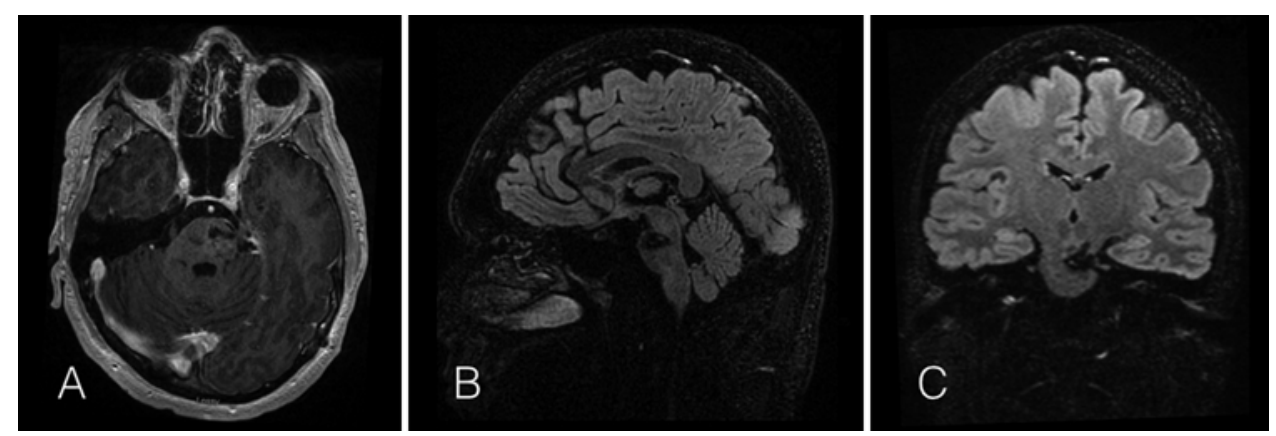

FIG. 6. Case 3. Postoperative axial T1-weighted postcontrast (A), and sagittal (B) and coronal T2-weighted CUBE-FLAIR (C) MR images show no obvious residual $\mathrm{CM}$ and significant improvement of mass effect. 

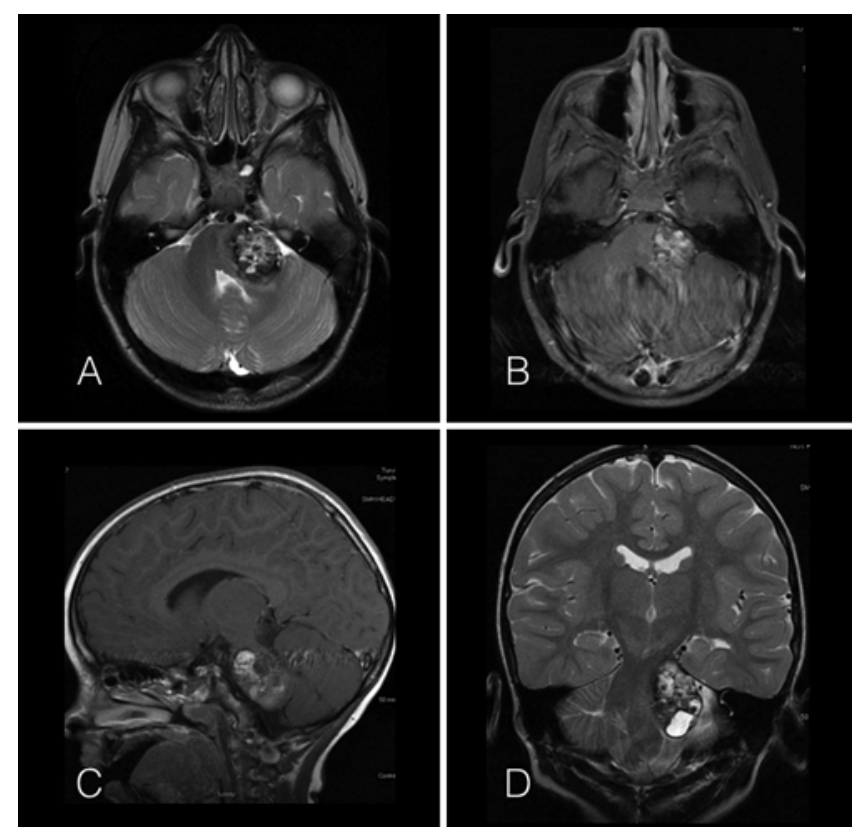

FIG. 7. Case 4. Preoperative axial T2-weighted (A), axial T1-weighted postcontrast (B), sagittal T1-weighted postcontrast (C), and coronal T2-weighted (D) MR images show a very large hemorrhagic contrastenhancing $\mathrm{CM}$ involving the left lateral pons and cerebellar peduncle at the level of the left cerebellopontine angle.

rhage is estimated to be as high as $60 \%$. In a case series consisting of 58 patients presenting with spontaneous brainstem hemorrhages, no patient with a Glasgow Coma Scale (GCS) score of 4 or less survived with conservative management and/or EVD placement. ${ }^{12}$

In adults, hypertension is by far the most common cause of spontaneous brainstem hemorrhages; however, anticoagulation therapy, amyloid angiopathy, tumor apoplexy, infections, arteriovenous malformations, and CMs also present similarly., ${ }^{917}$ General practice in the management of brainstem hemorrhages has been medical and conservative. This is due, in part, to the overall poor natural history and lack of a proven and effective surgical intervention. On the other hand, in young healthy individuals presenting with brainstem hemorrhages, an underlying lesion should be considered, and MRI can be helpful in detecting an underlying vascular malformation as well as defining the anatomy in case emergency surgery is indi- cated. In a recent meta-analysis, $35 \%$ of patients diagnosed with CMs had a brainstem location. CMs in the brainstem have a distinctly higher risk of intracerebral hemorrhage or focal neurological deficits compared with CMs in other locations, with the latter presenting more commonly with seizures or headaches. CMs in the brainstem were independently associated with poorer prognosis and were classified as the highest risk group of patients with CMs. ${ }^{6}$ Patients with brainstem CMs are also very likely to rehemorrhage soon after an initial event, thereby resulting in new or more pronounced deficits. These relapsing and remitting symptoms can become catastrophic; therefore, obtaining an early MR image can be valuable if clinical suspicion is high.

Most patients with brainstem CMs present with suddenonset $\mathrm{CN}$ deficits or ataxia, but uncommonly patients can present in extremis from an acute hemorrhage. In rapidly decompensating patients, urgent surgery can be lifesaving. Therefore, it is important to suspect brainstem CMs in young patients presenting with a brainstem hemorrhage and to obtain MRI to identify these lesions.

Surgery has long been accepted as the treatment of choice for CMs, but because of the surgical risks of approaching the brainstem, radiosurgery has also been increasingly evaluated as a therapeutic alternative. Although no treatment guidelines exist in the management of brainstem CMs, the natural history suggests they have a high risk of functional neurological deficits, therefore lending strong support toward intervention.

The timing of surgery for brainstem CMs has been a controversial topic. On the one hand, surgery can result in symptom recovery and functional protection, while on the other, cerebral edema and acute hematoma can distort lesional and normal anatomical boundaries, making surgery more difficult. Many authors propose delaying surgery into the subacute phase when perilesional edema has subsided, hematoma has liquefied, and neurological function has stabilized, thereby facilitating surgical decision making. ${ }^{8}$ When surgery is delayed until the chronic stage, scar tissue and adhesions can become prominent, making surgical dissection more tedious. ${ }^{11,16}$

In cases of rapidly progressive neurological deterioration, emergency resection is often the only option. A previous review of 139 patients presenting with hemorrhagic brainstem CMs in which the conditions of 9 patients deteriorated to a GCS score of 3, suggests that the natural
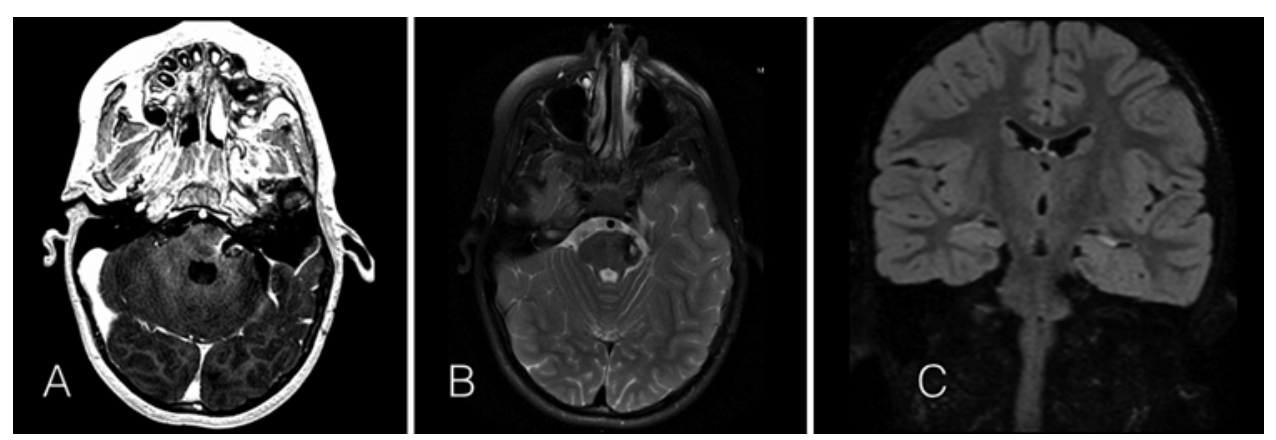

FIG. 8. Case 4. Postoperative axial T2-weighted (A), axial T1-weighted postcontrast (B), and coronal T2-weighted (C) MR images show no obvious residual $\mathrm{CM}$ and preserved venous anomaly adjacent to the resection bed $(\mathrm{A})$. 


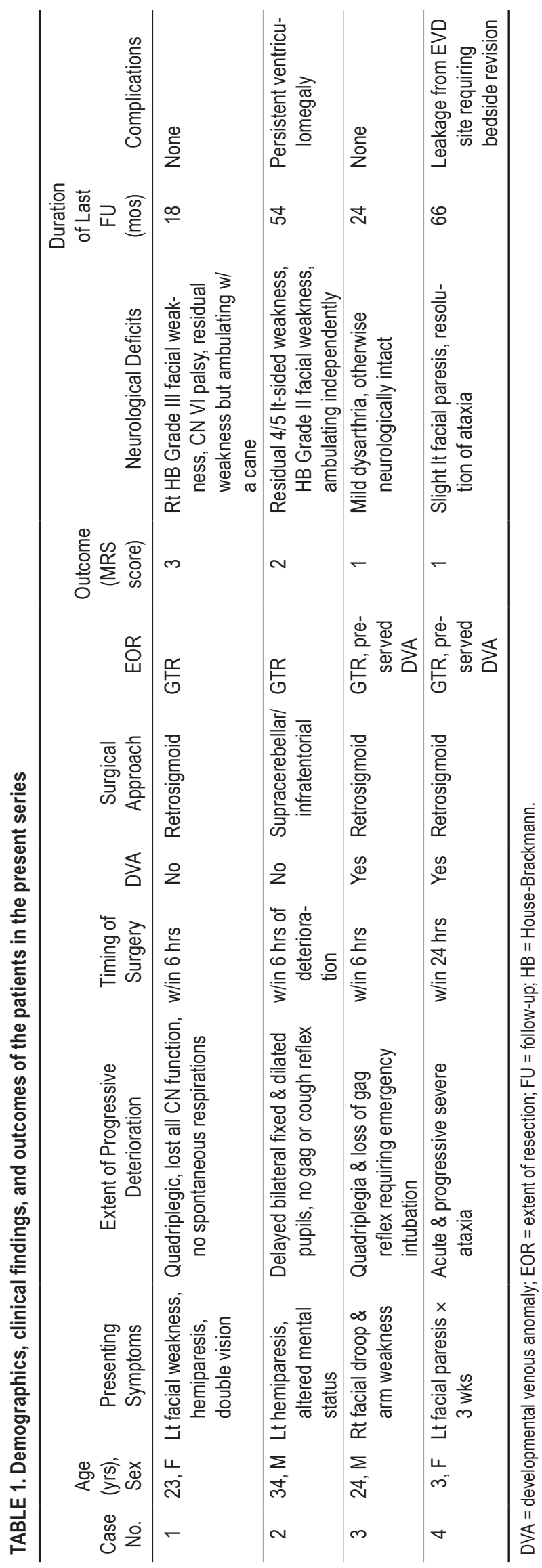

history is bleak, as none survived with conservative therapy alone. ${ }^{3}$ Our case series suggests that surgery can be performed safely and result in significant early recovery of severe neurological deficits in an emergency setting. In the 2 largest published series reporting on surgical outcomes of brainstem CMs, no mention is made of the urgent or emergent timing of surgery. This could be attributed to a lack of surgical consideration, which is the conventional management strategy in patients presenting in extremis with brainstem hemorrhages due to the purported poor natural history. ${ }^{1,3,10}$

To the best of our knowledge, we have now reported the first surgical experience focusing on emergency surgery for the treatment of brainstem CMs. Emergency surgery (within 24 hours) for brainstem CMs in a rapidly deteriorating patient has been described in the literature, but little is published specifically regarding the functional outcomes in these cases; therefore, unfortunately, no additional case details can be accrued to this report. ${ }^{2,16}$ Based on our research, only 1 other author has reported on outcomes after resection for patients after rapidly progressive neurological deterioration. Sindou et al. ${ }^{14}$ reported on a series of 12 patients who underwent delayed resection for brainstem CMs in which 2 of the 12 patients were comatose after a second rehemorrhage, deteriorating to a presurgical Karnofsky Performance Scale (KPS) score of 20. Although the timing of surgery from the onset of rapid deterioration is not reported, both patients improved postoperatively at the 1-year follow-up, with KPS scores of 50 and 90.

In our perusal of the literature, we did encounter a published experience of 5 patients presenting with hypertensive brainstem hemorrhages who underwent emergency surgical decompression and hematoma evacuation. These patients presented with GCS scores between 3 and 7. No patient recovered to a modified Rankin Scale (mRS) score of 1 , but 2 of 5 patients initially presenting with GCS scores of 3 and 5 had improved to an mRS score of 3, suggesting that acute amelioration of mass effect may have a role in symptom recovery (Table 2$) \cdot{ }^{13}$ On the other hand, these outcomes contrast with our own experience showing only modest improvements after emergency surgery (Table 1). The difference in pathophysiology and a more substantial neurological improvement after surgery for brainstem CMs may be attributed to the splaying of fiber tracts toward the pial surface and a larger operative cavity created by the hemorrhagic lesion, which facilitates a safer resection. Hypertensive hemorrhages, on the other hand, may result in greater irreparable destruction of the brainstem parenchyma, thereby contributing to poorer outcomes despite early and decompressive surgery.

Brainstem CMs are focal lesions with distinct anatomical boundaries from the surrounding cerebral tissue analogous to a mass lesion such as tumor or infection. The importance of this distinction has been supported by our case series because emergency surgery can be lifesaving. Our series presents preliminary evidence that some "lesional" (vascular malformation or neoplasm) brainstem hemorrhages in patients with severe and rapidly progressive deficits can be treated safely in the acute setting, with significant early recovery of even devastating neurological deficits. This result challenges the traditional management 
TABLE 2. Five cases of patients with hypertensive brainstem hemorrhages in the literature

\begin{tabular}{|c|c|c|c|c|c|}
\hline Case No. & Age (yrs) & Presenting Symptoms* & Timing of Surgery & Surgical Approach & Outcome (mRS Score) \\
\hline 1 & 44 & Comatose, sluggish pupils, GCS score 3 & w/in 2 hrs & Subtemporal & 3 \\
\hline 2 & 37 & Facial nerve palsy, pinpoint pupils, unreactive, GCS score 5 & Emergently & Suboccipital midline & 3 \\
\hline 3 & 70 & Comatose, GCS score 4 & Emergently & Retrosigmoid & 5 \\
\hline 4 & 59 & Anisocoria, absent light reflex, tetraplegia, GCS score 6 & Emergently & Subtemporal & 5 \\
\hline 5 & 42 & Quadriparesis, aphasia, blurry vision, GCS score 7 & Emergently & Suboccipital midline & 5 \\
\hline
\end{tabular}

Based on data from Shrestha et al. ${ }^{13}$

* The conditions of all 5 of these patients did not progress, in contrast to the patients in our series, in which some did.

strategy. Lesional brainstem hemorrhages could potentially be considered a distinct entity within the management paradigm, but further studies are needed to determine if an aggressive treatment approach to these lesions would be more optimal than conservative regimens.

A number of surgical strategies and safe entry zones have been described to approach brainstem lesions. The surgeon should use the approach that is the safest and most familiar in his or her hands based on the specific anatomical features of each lesion. The surgeon should keep in mind that local mass effect will often distort normal anatomical landmarks; therefore, neuromonitoring can be an important surgical adjunct. In our experience, during emergency surgery we did not encounter any significant difficulty due to brainstem edema, and overall surgery was not significantly more difficult compared with our other more elective cases. None of the patients presented here experienced worsening of these neurological deficits or near addition of deficits.

A recent grading system for brainstem $\mathrm{CMs}$ has been proposed by Garcia et al., ${ }^{4}$ highlighting the prognostic importance of size, location, presence of a developmental venous anomaly, age, and the timing of surgery after hemorrhage on outcomes after surgery of brainstem CMs. The authors recommend surgery in an "acute" setting (within 3 weeks) after a recent hemorrhage, citing stabilization of the patient's condition by reducing perilesional edema and capitalizing on liquefied hematoma as potential contributors to improved patient outcomes. Although the authors do not comment on emergency surgery for patients with severe neurological deficits, their proposed outcome predictors are important when weighing the risks of surgery against the expected surgical results. The indications for emergency surgery should be individualized based on the patient's baseline functional status, comorbid systemic disease, age, and outcome goals.

The main limitations of this study include its small sample size and the unknown natural history of these cases had conservative management or delayed surgery been used. Hemorrhagic brainstem CMs will often have a relapsing remitting course, and neurological deficits frequently improve after an acute hemorrhagic event; therefore, it is uncertain if these patients would have had similar outcomes had surgery been delayed or deferred. In patients with mild to moderate neurological deficits we would err on the conservative side; however, we would argue in cases of severe life-threatening neurological decline, as demonstrated in at least 3 of our cases, that we do not have the luxury of expectant management. Especially in patients who are comatose, the general consensus is that the natural history is poor with conservative management. We have been aggressive in our management of these patients and present a small series of better than expected results. We would not recommend withholding a potential life-saving intervention; therefore, the true natural history of rapidly decompensating hemorrhagic brainstem CMs may remain elusive.

In summary, we present favorable outcomes of patients presenting in extremis with hemorrhagic brainstem CMs after undergoing emergency surgery. In rapidly decompensating patients, emergency surgery can be lifesaving. Young, healthy individuals presenting with brainstem hemorrhages should raise suspicion for an underlying lesion, and MRI should be considered because early and significant reversal of neurological deficits can be observed with emergency decompression.

\section{References}

1. Abla AA, Lekovic GP, Turner JD, de Oliveira JG, Porter R, Spetzler RF: Advances in the treatment and outcome of brainstem cavernous malformation surgery: a single-center case series of 300 surgically treated patients. Neurosurgery 68:403-415, 2011

2. Bruneau M, Bijlenga P, Reverdin A, Rilliet B, Regli L, Villemure JG, et al: Early surgery for brainstem cavernomas. Acta Neurochir (Wien) 148:405-414, 2006

3. Fritschi JA, Reulen HJ, Spetzler RF, Zabramski JM: Cavernous malformations of the brain stem. A review of 139 cases. Acta Neurochir (Wien) 130:35-46, 1994

4. Garcia RM, Ivan ME, Lawton MT: Brainstem cavernous malformations: surgical results in 104 patients and a proposed grading system to predict neurological outcomes. Neurosurgery 76:265-278, 2015

5. Hauck EF, Barnett SL, White JA, Samson D: Symptomatic brainstem cavernomas. Neurosurgery 64:61-71, 2009

6. Horne MA, Flemming KD, Su IC, Stapf C, Jeon JP, Li D, et al: Clinical course of untreated cerebral cavernous malformations: a meta-analysis of individual patient data. Lancet Neurol 15:166-173, 2015

7. Li D, Hao SY, Jia GJ, Wu Z, Zhang LW, Zhang JT: Hemorrhage risks and functional outcomes of untreated brainstem cavernous malformations. J Neurosurg 121:32-41, 2014

8. Menon G, Gopalakrishnan CV, Rao BR, Nair S, Sudhir J, Sharma M: A single institution series of cavernomas of the brainstem. J Clin Neurosci 18:1210-1214, 2011

9. Murata Y, Yamaguchi S, Kajikawa H, Yamamura K, Sumioka S, Nakamura S: Relationship between the clinical manifestations, computed tomographic findings and the outcome in 80 patients with primary pontine hemorrhage. J Neurol Sci 167:107-111, 1999

10. Pandey P, Westbroek EM, Gooderham PA, Steinberg GK: 
Cavernous malformation of brainstem, thalamus, and basal ganglia: a series of 176 patients. Neurosurgery 72:573-589, 2013

11. Ramina R, Mattei TA, de Aguiar PH, Meneses MS, Ferraz VR, Aires R, et al: Surgical management of brainstem cavernous malformations. Neurol Sci 32:1013-1028, 2011

12. Rohde V, Berns E, Rohde I, Gilsbach JM, Ryang YM: Experiences in the management of brainstem hematomas. Neurosurg Rev 30:219-224, 2007

13. Shrestha BK, Ma L, Lan ZG, Li H, You C: Surgical management of spontaneous hypertensive brainstem hemorrhage. Interdisciplinary Neurosurg 2:145-148, 2015

14. Sindou M, Yada J, Salord F: Functional results after microsurgical resection of brain stem cavernous malformations (retrospective study of a 12 patient series and review of the recent literature). Acta Neurochir (Wien) 142:843-853, 2000

15. Taslimi S, Modabbernia A, Amin-Hanjani S, Barker FG II, Macdonald RL: Natural history of cavernous malformation: systematic review and meta-analysis of 25 studies. Neurology 86:1984-1991, 2016

16. Wang CC, Liu A, Zhang JT, Sun B, Zhao YL: Surgical management of brain-stem cavernous malformations: report of 137 cases. Surg Neurol 59:444-454, 2003

17. Ye Z, Huang X, Han Z, Shao B, Cheng J, Wang Z, et al:
Three-year prognosis of first-ever primary pontine hemorrhage in a hospital-based registry. J Clin Neurosci 22:11331138,2015

\section{Disclosures}

The authors report no conflict of interest concerning the materials or methods used in this study or the findings specified in this paper.

\section{Author Contributions}

Conception and design: Baskaya, Tumturk, Li. Acquisition of data: Tumturk, Li, Turan, Cikla. Analysis and interpretation of data: all authors. Drafting the article: Tumturk, Li, Turan, Cikla. Critically revising the article: Baskaya, Iskandar. Reviewed submitted version of manuscript: all authors. Approved the final version of the manuscript on behalf of all authors: Baskaya.

\section{Correspondence}

Mustafa K. Baskaya, Department of Neurological Surgery, University of Wisconsin-Madison, School of Medicine, CSC, K4/882, 600 Highland Ave., Madison, WI 53792.email: baskaya@ neurosurgery.wisc.edu. 